\title{
Prognostic significance and predictors of the neutrophil-to-lymphocyte ratio in ovarian cancer
}

\section{Citation}

Williams, Kristina A., S. Intidhar Labidi-Galy, Kathryn L. Terry, Allison F. Vitonis, William R. Welch, Annekathryn Goodman, and Daniel W. Cramer. 2014. Prognostic Significance and Predictors of the Neutrophil-to-Lymphocyte Ratio in Ovarian Cancer. Gynecologic Oncology 132, no. 3: 542-550. doi:10.1016/j.ygyno.2014.01.026.

\section{Published Version}

doi:10.1016/j.ygyno.2014.01.026

\section{Permanent link}

http://nrs.harvard.edu/urn-3:HUL.InstRepos:32303181

\section{Terms of Use}

This article was downloaded from Harvard University's DASH repository, and is made available under the terms and conditions applicable to Open Access Policy Articles, as set forth at http:// nrs.harvard.edu/urn-3:HUL.InstRepos:dash.current.terms-of-use\#OAP

\section{Share Your Story}

The Harvard community has made this article openly available.

Please share how this access benefits you. Submit a story.

\section{Accessibility}




\title{
Prognostic Significance and Predictors of the Neutrophil-to- Lymphocyte Ratio in Ovarian Cancer
}

\author{
Kristina A. Williams ${ }^{1,2}$, S. Intidhar Labidi-Galy ${ }^{3}$, Kathryn L. Terry ${ }^{1,2,4}$, Allison F. Vitonis ${ }^{1}$, \\ William R. Welch ${ }^{5}$, Annekathryn Goodman ${ }^{2,6}$, and Daniel W. Cramer ${ }^{1,2,4}$
}

${ }^{1}$ Obstetrics and Gynecology Epidemiology Center, Department of Obstetrics and Gynecology, Brigham and Women's Hospital, 221 Longwood Ave, Boston, Massachusetts $02115^{2}$ Harvard Medical School, 260 Longwood Avenue, Boston, Massachusetts $02115^{3}$ Dana Farber Cancer Institute, 44 Binney Street, Boston, MA; Current Affiliation: Center of Oncology, Geneva University Hospitals, Rue Gabrielle-Perret-Gentil 41205 Geneva, Switzerland and University of Geneva, Rue du Général- Dufour 241211 Geneva, Switzerland ${ }^{4}$ Department of Epidemiology, Harvard School of Public Health, 677 Huntington Ave., Boston, Massachusetts 02115 ${ }^{5}$ Department of Pathology, Brigham and Women's Hospital, 75 Francis Street, Boston, Massachusetts $02115^{6}$ Gillette Center for Women's Cancer, Massachusetts General Hospital, 55 Fruit Street, Boston, MA 02114

\section{Abstract}

Objective-To investigate the neutrophil-to-lymphocyte ratio (NLR) from peripheral blood, a general measure of inflammation, in ovarian cancer.

Methods-White cell counts and CA125 levels before treatment, tumor features, and questionnaire data on 519 women with ovarian cancer at two Boston hospitals were recorded. Counts were log-transformed and effects on these by tumor features and epidemiologic variables assessed by analysis of variance and generalized linear models. Cox proportional hazards models were used to assess effects on overall survival.

Results-Greater NLR was associated with higher tumor stage and grade, presence of ascites, and bilateral disease and correlated with risk factors including Jewish ethnicity, taller height, more ovulatory cycles, and family history of cancer in premenopausal women and talc use in all women. CA125 was positively correlated with neutrophil count, monocyte count, and NLR and inversely correlated with lymphocyte count. In a multivariate adjusted analysis, high NLR predicted poorer survival and high lymphocyte count better survival.

Conclusion-An elevated NLR before treatment signals more aggressive disease and correlates with risk factors for ovarian cancer. CA125 directly correlates with neutrophils which may reflect secretion of both CA125 and neutrophilic growth factors by the tumor. CA125 inversely correlates with lymphocytes which may reflect the ability of some neutrophilic factors to induce

(C) 2014 Elsevier Inc. All rights reserved.

Corresponding author: Daniel W. Cramer, M.D., Sc.D., Obstetrics and Gynecology Epidemiology Center, 221 Longwood Avenue, RFB 365, Brigham and Women's Hospital, Boston MA 02115, Tel: 617-732-4895, Fax: 617-732-4899, dcramer@partners.org.

Publisher's Disclaimer: This is a PDF file of an unedited manuscript that has been accepted for publication. As a service to our customers we are providing this early version of the manuscript. The manuscript will undergo copyediting, typesetting, and review of the resulting proof before it is published in its final citable form. Please note that during the production process errors may be discovered which could affect the content, and all legal disclaimers that apply to the journal pertain.

Potential Conflicts of Interest

Dr. Cramer reports being paid for expert testimony in litigation related to ovarian cancer. No other disclosures are reported. 
lymphopenia and/or binding of CA125 to lymphocytes removing CA125 from the serum pool. Links between NLR, CA125, and epidemiologic factors may provide new clues about the pathogenesis and progression of ovarian cancer.

\section{Keywords}

Ovarian Cancer; Risk Factors; CA125

\section{Introduction}

A general measure of inflammation and oxidative stress in peripheral blood is the ratio of neutrophils to lymphocytes (NLR)--not to be confused with another NLR in immune jargon, NOD-like receptors[1]. The neutrophil to lymphocyte ratio correlates with disease severity and prognosis for many different malignant and non-malignant diseases. [2]. [3] Diseasepredisposing conditions, like obesity, the metabolic syndrome, and smoking are also associated with elevated NLR; and both the NLR and disease risk can be lowered by weight loss or smoking cessation.[4][5][6][7] These observations suggest the NLR is not simply a reflection of end-stage illness but may be involved in disease pathogenesis as well.

For ovarian cancer, NLR measured before diagnosis can identify CA125-negative cases and is more sensitive than CA125 in predicting survival.[8] That an elevated NLR predicts ovarian cancer prognosis is not surprising since both the neutrophil count and the lymphocyte count individually correlate with survival but in opposite directions. A higher neutrophil count, as well as total white blood cell count (WBC), and a lower lymphocyte count predict poorer survival.[8][9][10][11][12] That CA125 may bind to certain leukocyte sub-populations suggests a relationship between NLR and CA125 that is much stronger than the two simply being complementary markers.[13] In this study, we have conducted a more detailed examination of pre-operative WBC, differential counts, and the NLR in women with ovarian cancer and their correlations with tumor features, CA125, epidemiologic factors, and overall survival.

\section{Methods \\ Case population}

Women with ovarian cancer in this study were enrolled from two protocols—one that recruits patients before surgery for a pelvic mass and a second after a diagnosis of cancer has already been made. Details regarding these protocols have been previously described. [14] Briefly, both protocols are approved by the Brigham and Women's Hospital (BWH) and Massachusetts General Hospital (MGH) Institutional Review Boards allowing for questionnaires, specimen collection, and medical record reviews. The pelvic mass study recruited subjects from Gynecologic Oncology Clinics at MGH and the BWH/Dana Farber Cancer Institute (DFCI). Although the case-control study recruited cases from throughout eastern Massachusetts and New Hampshire, only patients diagnosed at BWH/DFCI and MGH were included for comparability to the pelvic mass group and feasibility in retrieving CA125 and WBC counts. Between 1992 and 2013, 455 cases from the pelvic mass study and 1,029 cases from the case-control study were enrolled from the three hospitals.

\section{Questionnaire and medical record data}

Patients enrolled in the pelvic mass study completed self-administered questionnaires and those enrolled in the case-control study completed longer questionnaires administered by study staff. Demographic data, reproductive history, key exposures, and medical and family history were collected. Medical records and computerized laboratory reports were reviewed 
to determine whether CA125 and WBC had been obtained prior to any therapy. We were able to obtain a preoperative CA125 within one month of the date of diagnosis for 305 $(67 \%)$ of the pelvic mass cases and $559(51 \%)$ of the case-control cases. Among these, 519 ovarian cancer cases [187 (61\%) from the pelvic mass cases and $332(59 \%)$ from the casecontrol study] also had a WBC within 14 days of the CA125 and prior to therapy. Age and histologic distributions were similar for cases with and without CA125 and WBC values available for analysis. Tumor characteristics, presence of ascites, and optimal debulking status (when available) were recorded from the operative note or preoperative imaging. For assessing overall survival, date of death was obtained from the chart or querying the Social Security Death Index. Follow-up data was insufficient to calculate disease free survival.

Details from the pathology report were abstracted including histologic type, stage, grade, and laterality of the tumor. Histological subtype classification was reviewed for consistency by a single pathologist (WRW). Subtypes included serous borderline, mucinous borderline, serous invasive, mucinous invasive, endometrioid, and clear cell tumors. Transitional cell tumors or mixed serous and transitional tumors were counted as serous as were undifferentiated tumors. Mixed tumors described as "predominantly" one type or containing "focal" area of another were coded to the predominant type. Mixed endometrioid and clear cell tumors were included with endometrioid. Other mixed epithelial tumors, malignant Brenner tumors and unspecified epithelial were grouped as "other".

\section{Statistical Methods}

Geometric means (GM) and 95\% confidence intervals (CI) for WBC, differential counts, and NLR in all ovarian cancer cases were calculated by enrollment features, tumor characteristics, and epidemiologic variables and compared by t tests, analysis of variance (ANOVA), or generalized linear models. Generalized linear models were used to compare epidemiologic variables with log-transformed WBC, differential counts, or NLR and all models were adjusted for histologic subtype, year of enrollment, and hospital. P-values for categorical (pcat) and trend (ptrend) variables were calculated with partial sum of squares Ftests. Trend tests were performed by modeling the medians of exposure categories as continuous variables. To examine potential effect modification by menopausal status, we performed likelihood ratio tests where a model including each exposure of interest and menopausal status was compared to a model with these terms and the interaction term between them. Spearman correlations of CA125 with total WBC and differential counts were calculated for all ovarian cancers and subtypes. Cox proportional hazard models (HR) were used to examine the association between CA125, WBC, differential counts, NLR and overall survival, adjusting for age, hospital, year of enrollment, histology, grade, stage, debulking status, smoking status, and BMI and, in a secondary model, additionally adjusting for CA125. A Wald test of the interaction term was used to calculate a p-value for interaction between each WBC parameter and CA125. All analyses were performed using SAS v9.2 (SAS Institute Inc., Cary, NC, USA).

\section{Results}

GMs for WBC, differential counts, and NLR by enrollment features and tumor characteristics are shown in Table 1. Study type and year or site of enrollment did not influence neutrophil and lymphocyte counts or the NLR but year of enrollment influenced the monocyte count and hospital the basophil count. In general, lymphocyte counts were significantly lower and neutrophil counts and NLR significantly higher with more aggressive tumor features including higher tumor grade and stage, presence of ascites, and bilateral disease. Invasive serous and mucinous tumors had lower lymphocyte counts and higher neutrophil counts and NLR compared to their borderline counterparts. There were significant differences in means by histologic type of ovarian cancer for; total WBC, 
neutrophils, lymphocytes, monocytes, and NLR. For cases enrolled in the pelvic mass study and those in the most recent phase of the case-control study, pathologic review included greater effort to identify coexisting intraepithelial tubal neoplasia suggesting serous tumors with possible fallopian tube origin. NLR was greatest for those with primary peritoneal tumors and least for those with a likely tubal origin of their cancer.

Effects of epidemiologic factors on mean levels of WBC, differential counts, and NLR are shown in Table 2. Jewish women had higher lymphocyte counts. Women who were heavier and had greater BMIs had higher total WBC, neutrophil, and monoctye counts, although the trend was not monotonic with women in the lowest and highest BMI and weight categories having higher counts than those in intermediate categories. Total WBC, neutrophils, lymphocyte, and basophil counts were significantly increased in current smokers and those with a greater number of pack-years. Lymphocyte counts were lower in women with 3 or more livebirths. Women with long term use of talc in the genital area had an elevated NLR. Some notable findings of borderline significance $(0.05>\mathrm{p}<0.10)$ included greater NLR with taller height, lower NLR with history of appendectomy, and lower WBCs and neutrophils with a history of puerperal mastitis.

Although menopausal status per se had no significant effects on WBC, differential counts, and NLR, we examined the associations in Table 2 stratified by menopausal status and found that the associations with several epidemiologic factors were more apparent when restricted to pre-menopausal women (Table 3). Total WBC and neutrophil counts were significantly elevated in premenopausal women who were Jewish, heavier, and had a greater number of estimated ovulatory cycles. Lymphocyte counts were higher in heavier premenopausal women and the NLR was elevated in premenopausal women who were taller and had a greater number of ovulatory cycles. Premenopausal women who had a family history of breast or ovarian cancer had lower lymphocyte counts and greater NLR.

Mean CA125 levels and correlations between CA125 and WBC, differential counts, and NLR are shown in Table 4. Among all cases, CA125 was positively correlated with total white blood count $(r=0.24, p<0.0001)$, neutrophil count $(r=0.32, p<0.0001)$, monocyte count $(\mathrm{r}=0.24, \mathrm{p}<0.0001)$, and NLR $(\mathrm{r}=0.42, \mathrm{p}<0.0001)$, whereas an inverse correlation was observed with lymphocytes $(\mathrm{r}=-0.27, \mathrm{p}<0.0001)$. These overall correlations largely reflect those found for invasive serous tumors-the predominant histologic type. However, positive (and generally significant) correlations between CA125 and the NLR were noted for each histologic type of ovarian cancer-being weakest for endometrioid tumors $(r=0.25, p=0.07)$ and strongest for clear cell tumors ( $\mathrm{r}=0.59, \mathrm{p}=0.001$ ). Clear cell tumors were the only tumor type for which CA125 was significantly correlated (inversely) with basophils $(r=-0.41$, $\mathrm{p}=0.04)$ and eosinophils $(\mathrm{r}=-0.40, \mathrm{p}=0.04)$.

Among the 519 cases in this study, 224 died and the median follow-up time was 5.7 years (range 1 month to 21 years). The influences of WBC, differential counts, and NLR on overall survival are shown in Table 5. After adjustment for age, hospital, year of enrollment, histologic subtype, grade, stage, optimal debulking status, smoking status, and BMI, predictors of poorer survival included higher CA125 (HR=1.10 95\%CI: 1.00-1.20, $\mathrm{p}=0.05$ ), and NLR (HR=1.43 95\%CI: 1.13-1.81, $\mathrm{p}=0.003$ ), whereas higher lymphocyte counts predicted better survival (HR=0.56 95\%CI: $0.40-0.80, \mathrm{p}=0.001$ ). Lymphocyte count and NLR persisted as significant predictors of survival after adjustment for CA125 and when cases were restricted to serous invasive (lymphocyte $\mathrm{HR}=0.6095 \% \mathrm{CI}: 0.42-0.85, \mathrm{p}=0.005$; NLR HR $=1.37$ 95\% CI: 1.06-1.76, $\mathrm{p}=0.02$, data not shown). Interactions between CA125 and WBC parameters were not found to affect survival (data not shown). 


\section{Discussion}

We examined pre-treatment WBC, differential counts, and the NLR among ovarian cancer cases in relation to tumor characteristics, epidemiologic factors, presenting CA125, and survival. Tumors with higher stage and grade were associated with higher NLR which, in turn, predicted poorer survival. Differential counts and NLR levels were also associated with epidemiologic factors such that, generally, factors that increase ovarian cancer risk were associated with higher NLR and factors that decrease risk with lower NLR. CA125 levels were inversely correlated with lymphocyte counts and positively correlated with neutrophil counts and the NLR. How these findings are supported by existing literature and what new insights about ovarian cancer they may contribute are discussed.

For many types of cancer, it is well established that an elevated NLR reflects more advanced stages of disease and larger tumors with more aggressive behavior.[3] For ovarian cancer, data on NLR is more limited. Cho et all found that NLR levels, measured before diagnosis, can identify CA125-negative cases and is more sensitive than CA125 in predicting both overall and disease-free survival.[8] Levels were also significantly elevated in ovarian cancer cases compared to those with benign gynecologic diseases or healthy controls. More studies have been done in relation to differential counts and ovarian cancer severity or prognosis. A higher neutrophil count, as well as total white blood cell count (WBC), and a lower lymphocyte count predict poorer survival.[8][9][10][11][12] Notably, Milne et al. found that ovarian cancer cases had significantly decreased lymphocytes at diagnosis compared to lymphocyte levels measured two years prior to diagnosis and that the decline was greater in women with higher stage, ascites, and suboptimal debulking.[11] In agreement, we found significantly lower lymphocyte counts and higher neutrophil counts and NLR associated with greater tumor grade and stage, presence of ascites, and bilateral disease. The correlation between NLR and tumor aggressiveness at least partially explains why NLR correlates strongly with survival after cancer. Despite the evidence that NLR is a robust predictor of survival after many different cancers including ovarian, larger studies with greater detail on patient profiles, tumor features, and treatment will be necessary to demonstrate that the NLR is truly an independent predictor.

Besides tumor features, we found differential counts and the NLR correlated with several epidemiologic factors. Increasing age is a strong risk factor for cancer in general and is associated with decreasing lymphocytes.[15] However, in our study restricted to ovarian cancer cases, we did not see a clear correlation with age. Jewish women, especially if they were premenopausal, had higher WBC and neutrophil counts. Women with a BMI >30 had higher total WBC, neutrophil, and lymphocyte counts. Current smokers, especially those who smoked more, had higher total WBC, neutrophils, and basophils. BMI and smoking are known to produce pro-inflammatory states in which WBC, lymphocyte, neutrophil, and monocyte counts--all increase with increasing BMI or pack-years of smoking.[16][17] Smoking cessation and weight loss are each associated with reduction of the leukocyte count and other measures of oxidative stress and inflammation.[6][7]

We observed lower lymphocytes and higher NLR among women of taller height. Height has been shown to be a factor that independently increases risk for various cancers in women, [18] including ovarian.[19] Genetic factors might play a role in these associations since about $80 \%$ of adult height may reflect inheritance.[20] One of the more interesting genes associated with height, especially in women, is LIN28B which is also associated with age at menarche.[21][22] Overexpression of LIN28B in transgenic mice leads to inflammatory cytokine production and increased c-reactive protein.[23] The positive correlation between height and NLR warrants further study looking for links with inflammation. 
A greater number of estimated ovulatory cycles has consistently been found to increase ovarian cancer risk.[24] We found that neutrophil counts and the NLR increased with a greater number of ovulatory cycles, especially among premenopausal ovarian cancer cases. This would be consistent with the observation that a greater number of ovulatory cycles is an especially strong risk factor for premenopausal ovarian cancer.[25] Long term use of talc use in genital hygiene is another factor consistently found to be associated with greater risk for ovarian cancer [26] and was associated with an elevated NLR in our study.

Premenopausal women who had a family history of breast or ovarian cancer also had an elevated NLR as well as a lower lymphoctye count. Conversely, two findings of borderline significance were that oral contraceptive use in premenopausal women was associated with lower NLR, as was history of puerperal mastitis. The latter finding is of interest because we recently reported that puerperal mastitis may be associated with lower risk for ovarian cancer possibly through its ability to lead to anti-CA125 antibodies or antibodies against another mucin CA15.3.[27] These antibodies may serve in immune surveillance to reduce risk for ovarian cancer. Thus, we have postulated that certain acute inflammatory events lead to protective anti-mucin antibodies and lower ovarian cancer risk whereas certain chronic inflammatory events lead to immune tolerance, lower anti-mucin antibodies, and increased risk for ovarian cancer which is mucinexpressing.[28][29][30] Observations from this study that the same epidemiologic factors also influence NLR levels now provides a link with cellular immunity that had been lacking in our data based solely on humoral immunity - a link revealed by correlations between the NLR and CA125.

A correlation between NLR and CA125 has been described for endometriosis[31] but not ovarian cancer. In our study, CA125 was positively correlated with total white blood count, neutrophil count, monocyte count, and NLR, whereas an inverse correlation was found for lymphocytes. Similar correlations with CA125 were seen for most histologic types of ovarian cancer, but not all were significant. An inverse correlation between the lymphocyte count and CA125 was not found for endometrioid ovarian cancer which also had the weakest positive correlation with NLR. Subtype differences in these correlations are potentially important because they may reflect the types and levels of the (tumor) antigens being produced by each specific tumor subtype. Earlier we noted that the NLR is positively correlated with tumor mass. A larger tumor mass both produces more CA125 and growth factors, like CSF-I, stem cell factors, and certain cytokines like IL-10 that would enhance neutrophil production in proportion to CA125[31][32][33] and lead to the positive correlation with neutrophils. However, IL-10 while inducing a neutrophilia, also causes lymphopenia.[34] The latter phenomenon, combined with the fact that some fraction of CA125 binds to certain lymphocytes and leaves the serum pool, are likely responsible for the negative correlation between lymphocytes and CA125. Binding of CA125 to lymphocytes may be mediated by the cell surface receptor, Siglec-9, and is capable of down regulating tumor immunogenicity. [13] Thus, CA125 appears to be much more than a passive tumor marker for ovarian cancer, but may actually be driving both its pathogenesis and progression through interaction with leukocytes. Lymphocyte count and CA125 binding might also explain why certain epidemiologic factors, like BMI and parity, might influence CA125 levels in both ovarian cancer cases and in the general population.[14]

Our interpretation of the observation that epidemiologic factors increasing risk for ovarian cancer also raised the NLR (e.g. tall stature, ovulatory cycles, use of talc in genital hygiene) is that the underlying inflammatory states associated with these factors are also capable of producing leukocyte stimulating and inhibitory factors just as the tumor mass itself. Links between inflammation and hematopoietic cell growth factors have been postulated by Hamilton.[35] These observations reinforce the role of the NLR as a general marker of oxidative stress, which may be equally important to its role as a prognostic factor. A better understanding of the connections between neutrophila, lymphopenia, NLR, and cancer risk 
factors may contribute new ideas regarding the pathogenesis, prevention, and early detection of ovarian cancer. This will require that additional comprehensive studies of the preoperative level of NLR in cases, as well as in healthy controls, be performed in order to determine whether the observations made here for ovarian cancer cases can be confirmed and extended to healthy women. If so, this would point to fundamental and important physiologic relationships that connect inflammation, immunity, leukocyte subpopulations, and CA125. These studies should be comprehensive in nature and also include measurement of another important mucin tumor antigen, CA15.3 (MUC1), as well as antibodies against both CA125 and CA15.3. An additional avenue of research would be to link peripheral counts and the NLR with lymphocytes and macrophages found in the tumors themselves.

Aside from the fact that our study was restricted to cases, there are other potential limitations. Because this study is the first to examine multiple epidemiologic features in ovarian cancer cases in relation to differential counts, chance is a possible explanation for some findings. Thus, of 154 associations explored in Table 2, about 8 significant associations might be expected by chance alone-- 18 were observed, most of which have prior support in the literature. A very conservative $\mathrm{p}$ value corrected for multiple testing would be about $\mathrm{p}=0.0003$ but using that cutoff comes at the expense of false negatives and does not ensure only valid associations are reported. Thus validation can only come from new studies looking at the NLR. Our study population is composed primarily of Caucasian women, so we are not able to generalize our results to other ethnic groups that may have different leukocyte profiles compared to Caucasians. Also, there is an obvious exception to our "rule" that factors that increase or decrease ovarian cancer operate in the opposite manner on the NLR. Higher parity lowers risk for ovarian cancer but, in our study, led to slightly lower lymphocyte counts and slightly higher NLR. It may be necessary to look at differences in lymphocyte subpopulations between nulliparous and multiparous women to clarify the nature of this exception. Such studies may inform how having children acts to lower risk for several cancers. Because we observed some variation in basophil and monocyte counts by year of study and hospital, laboratory variation may need to be standardized in future studies. However, we adjusted for enrollment features in analysis of the data related to neutrophil counts, lymphocyte counts, and the NLR.

In summary, we believe these are exciting new observations about the NLR and how it may correlate with both risk factors for ovarian cancer and CA125 expressed by those cancers. Confirmation of these findings in a new set of ovarian cases and confirming whether these associations pertain to healthy controls should be a high priority for ovarian cancer research and may have wide applicability to other cancers as well.

\section{Acknowledgments}

Funding: Supported by grants from the Department of Defense, W81XWH-10-1-0280 (Terry) and NCI, R01 CA054419 (Cramer).

\section{References}

1. Geddes K, Magalhaes JG, Girardin SE. Unleashing the therapeutic potential of NOD-like receptors. Nat Rev Drug Discov. 2009; 8:465-479. [PubMed: 19483708]

2. Bhat T, Teli S, Rijal J, Bhat H, Raza M, Khoueiry G, et al. Neutrophil to lymphocyte ratio and cardiovascular diseases: a review. Expert Rev Cardiovasc Ther. 2013; 11:55-59. [PubMed: 23259445]

3. Guthrie GJ, Charles KA, Roxburgh CS, Horgan PG, McMillan DC, Clarke SJ. The systemic inflammation-based neutrophil-lymphocyte ratio: Experience in patients with cancer. Crit Rev Oncol Hematol. 2013; 88:218-230. [PubMed: 23602134] 
4. Buyukkaya E, Karakas MF, Karakas E, Akcay AB, Kurt M, Tanboga IH, et al. Correlation of Neutrophil to Lymphocyte Ratio With the Presence and Severity of Metabolic Syndrome. Clin Appl Thromb Hemost. 2012 Sep 18. [Epub ahead of print].

5. Calapai G, Caputi AP, Mannucci C, Russo GA, Gregg E, Puntoni R, et al. Cardiovascular biomarkers in groups of established smokers after a decade of smoking. Basic Clin Pharmacol Toxicol. 2009; 104:322-328. [PubMed: 19175368]

6. Chae JS, Paik JK, Kang R, Kim M, Choi Y, Lee SH, et al. Mild weight loss reduces inflammatory cytokines, leukocyte count, and oxidative stress in overweight and moderately obese participants treated for 3 years with dietary modification. Nutr Res. 2013; 33:195-203. [PubMed: 23507225]

7. Van Tiel E, Peeters PH, Smit HA, Nagelkerke NJ, Van Loon AJ, Grobbee DE, et al. Quitting smoking may restore hematological characteristics within five years. Ann Epidemiol. 2002; 12:378388. [PubMed: 12160596]

8. Cho H, Hur HW, Kim SW, Kim SH, Kim JH, Kim YT, et al. Pre-treatment neutrophil to lymphocyte ratio is elevated in epithelial ovarian cancer and predicts survival after treatment. Cancer Immunol Immunother. 2009; 58:15-23. [PubMed: 18414853]

9. Webb MJ, Malkasian GD Jr, Jorgensen EO. Factors influencing ovarian cancer survival after chemotherapy. Obstet Gynecol. 1974; 44:564-570. [PubMed: 4413425]

10. Wolff JP, de Oliveira CF. Lymphocytes in patients with ovarian cancer. Obstet Gynecol. 1975; 45:656-658. [PubMed: 1143727]

11. Milne K, Alexander C, Webb JR, Sun W, Dillon K, Kalloger SE, et al. Absolute lymphocyte count is associated with survival in ovarian cancer independent of tumor-infiltrating lymphocytes. $\mathrm{J}$ Transl Med. 2012; 10:33. [PubMed: 22369276]

12. Bishara S, Griffin M, Cargill A, Bali A, Gore ME, Kaye SB, et al. Pre-treatment white blood cell subtypes as prognostic indicators in ovarian cancer. Eur J Obstet Gynecol Reprod Biol. 2008; 138:71-75. [PubMed: 17644243]

13. Belisle JA, Horibata S, Jennifer GA, Petrie S, Kapur A, Andre S, et al. Identification of Siglec-9 as the receptor for MUC16 on human NK cells, B cells, and monocytes. Mol Cancer. 2010; 9:118. [PubMed: 20497550]

14. Cramer DW, Vitonis AF, Welch WR, Terry KL, Goodman A, Rueda BR, et al. Correlates of the preoperative level of CA125 at presentation of ovarian cancer. Gynecol Oncol. 2010; 119:462468. [PubMed: 20850174]

15. De Paoli P, Battistin S, Santini GF. Age-related changes in human lymphocyte subsets: progressive reduction of the CD4 CD45R (suppressor inducer) population. Clin Immunol Immunopathol. 1988; 48:290-296. [PubMed: 2456868]

16. Schwartz J, Weiss ST. Cigarette smoking and peripheral blood leukocyte differentials. Ann Epidemiol. 1994; 4:236-242. [PubMed: 8055125]

17. Nieman DC, Henson DA, Nehlsen-Cannarella SL, Ekkens M, Utter AC, Butterworth DE, et al. Influence of obesity on immune function. J Am Diet Assoc. 1999; 99:294-299. [PubMed: 10076580]

18. Green J, Cairns BJ, Casabonne D, Wright FL, Reeves G, Beral V. Height and cancer incidence in the Million Women Study: prospective cohort, and meta-analysis of prospective studies of height and total cancer risk. Lancet Oncol. 2011; 12:785-794. [PubMed: 21782509]

19. Ovarian cancer and body size: individual participant meta-analysis including 25,157 women with ovarian cancer from 47 epidemiological studies. PLoS Med. 2012; 9:e1001200. [PubMed: 22606070]

20. Lettre G. Recent progress in the study of the genetics of height. Hum Genet. 2011; 129:465-472. [PubMed: 21340692]

21. Widen E, Ripatti S, Cousminer DL, Surakka I, Lappalainen T, Jarvelin MR, et al. Distinct variants at LIN28B influence growth in height from birth to adulthood. Am J Hum Genet. 2010; 86:773782. [PubMed: 20398887]

22. Ong KK, Elks CE, Li S, Zhao JH, Luan J, Andersen LB, et al. Genetic variation in LIN28B is associated with the timing of puberty. Nat Genet. 2009; 41:729-733. [PubMed: 19448623] 
23. Beachy SH, Onozawa M, Chung YJ, Slape C, Bilke S, Francis P, et al. Enforced expression of Lin28b leads to impaired T-cell development, release of inflammatory cytokines, and peripheral Tcell lymphoma. Blood. 2012; 120:1048-1059. [PubMed: 22723554]

24. Pelucchi C, Galeone C, Talamini R, Bosetti C, Montella M, Negri E, et al. Lifetime ovulatory cycles and ovarian cancer risk in 2 Italian case-control studies. Am J Obstet Gynecol. 2007; 196:83, e1-e7. [PubMed: 17240246]

25. Tung KH, Wilkens LR, Wu AH, McDuffie K, Nomura AM, Kolonel LN, et al. Effect of anovulation factors on pre- and postmenopausal ovarian cancer risk: revisiting the incessant ovulation hypothesis. Am J Epidemiol. 2005; 161:321-329. [PubMed: 15692075]

26. Terry KL, Karageorgi S, Shvetsov YB, Merritt MA, Lurie G, Thompson PJ, et al. Genital powder use and risk of ovarian cancer: a pooled analysis of 8,525 cases and 9,859 controls. Cancer Prev Res (Phila). 2013; 6:811-821. [PubMed: 23761272]

27. Cramer DW, Williams K, Vitonis AF, Yamamoto HS, Stuebe A, Welch WR, et al. Puerperal mastitis: a reproductive event of importance affecting anti-mucin antibody levels and ovarian cancer risk. Cancer Causes Control. 2013; 24:1911-1923. [PubMed: 23925696]

28. Cramer DW, Titus-Ernstoff L, McKolanis JR, Welch WR, Vitonis AF, Berkowitz RS, et al. Conditions associated with antibodies against the tumor-associated antigen MUC1 and their relationship to risk for ovarian cancer. Cancer Epidemiol Biomarkers Prev. 2005; 14:1125-1131. [PubMed: 15894662]

29. Pinheiro SP, Hankinson SE, Tworoger SS, Rosner BA, McKolanis JR, Finn OJ, et al. Anti-MUC1 antibodies and ovarian cancer risk: prospective data from the Nurses' Health Studies. Cancer Epidemiol Biomarkers Prev. 2010; 19:1595-1601. [PubMed: 20501761]

30. Terry KL, Titus-Ernstoff L, McKolanis JR, Welch WR, Finn OJ, Cramer DW. Incessant ovulation, mucin 1 immunity, and risk for ovarian cancer. Cancer Epidemiol Biomarkers Prev. 2007; 16:3035. [PubMed: 17220329]

31. Cho S, Cho H, Nam A, Kim HY, Choi YS, Park KH, et al. Neutrophil-to-lymphocyte ratio as an adjunct to CA-125 for the diagnosis of endometriosis. Fertil Steril. 2008; 90:2073-2079. [PubMed: 18555226]

32. Parrott JA, Kim G, Skinner MK. Expression and action of kit ligand/stem cell factor in normal human and bovine ovarian surface epithelium and ovarian cancer. Biol Reprod. 2000; 62:16001609. [PubMed: 10819761]

33. Pisa P, Halapi E, Pisa EK, Gerdin E, Hising C, Bucht A, et al. Selective expression of interleukin 10 , interferon gamma, and granulocyte-macrophage colony-stimulating factor in ovarian cancer biopsies. Proc Natl Acad Sci U S A. 1992; 89:7708-7712. [PubMed: 1502188]

34. Ponchel F, Verburg RJ, Bingham SJ, Brown AK, Moore J, Protheroe A, et al. Interleukin-7 deficiency in rheumatoid arthritis: consequences for therapy-induced lymphopenia. Arthritis Res Ther. 2005; 7:R80-R92. [PubMed: 15642146]

35. Hamilton JA. Colony-stimulating factors in inflammation and autoimmunity. Nat Rev Immunol. 2008; 8:533-544. [PubMed: 18551128] 


\section{Highlights}

- Peripheral blood neutrophil-to-lymphocyte ratio (NLR) is a general measure of inflammation

- NLR signals more aggressive disease and correlates with ovarian cancer risk factors and CA125

- These links with NLR may provide new clues about the pathogenesis and progression of ovarian cancer 
$\infty$

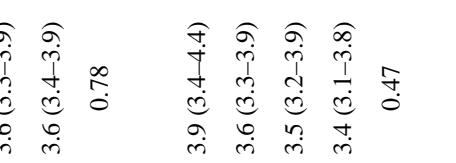

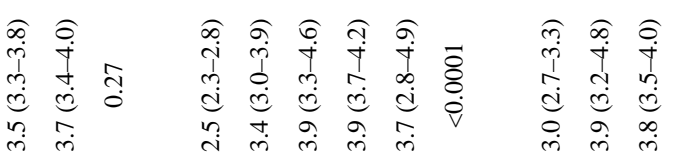

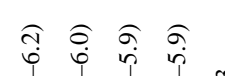
ڤั่

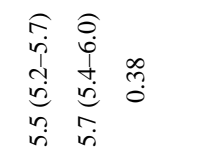

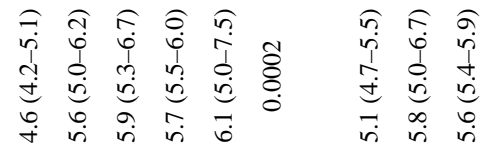




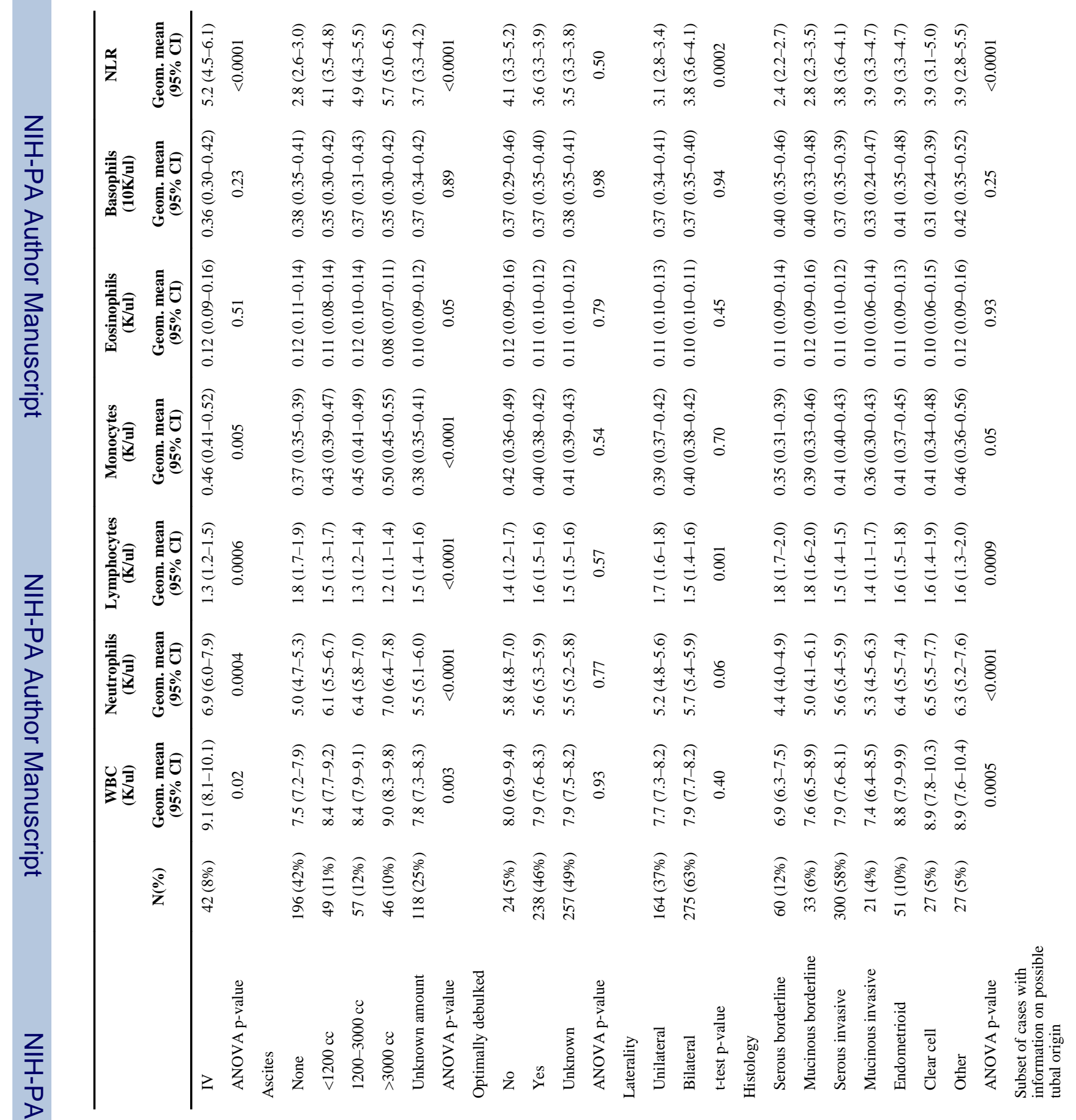




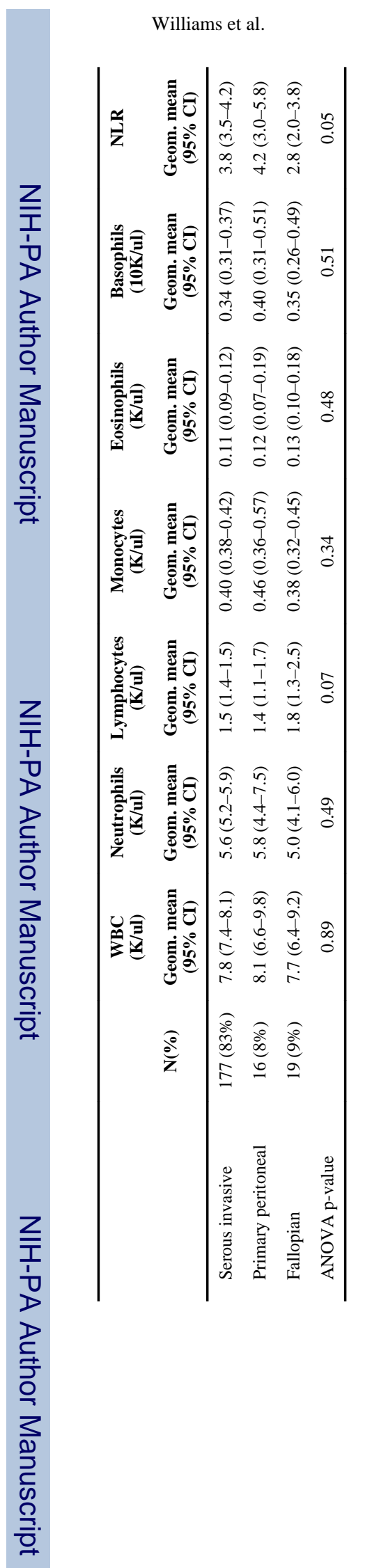

Gynecol Oncol. Author manuscript; available in PMC 2015 March 01. 


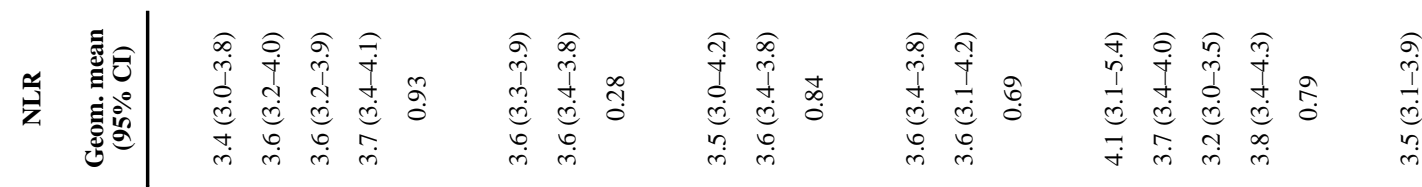

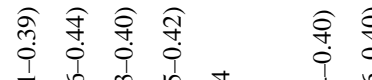

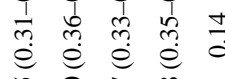

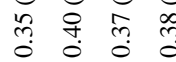

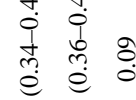

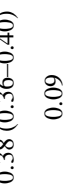

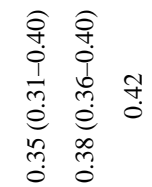

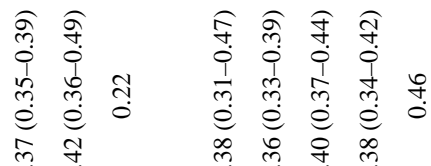

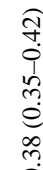

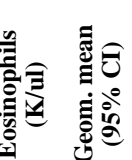

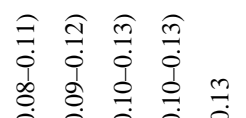

ิำำ

ำ

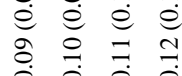

$\stackrel{0}{0} 0$

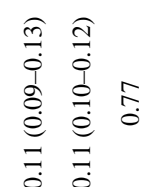

$\begin{array}{lll}\widehat{T} & \widehat{m} \\ 0 & 0 \\ 0 & 1 \\ 0 & 0 & 2 \\ 0 & 0 & 0 \\ 0 & 0 & 0 \\ = & 0 \\ 0 & 0\end{array}$

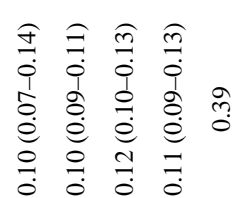

新㠻㠻

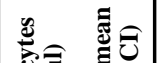

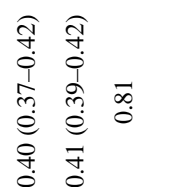

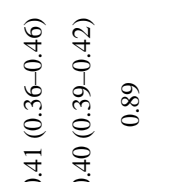

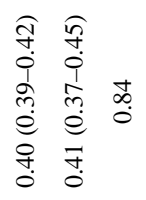

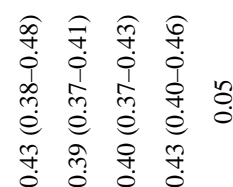

क.

I.

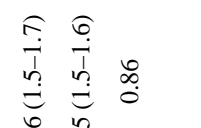

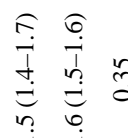

6. $\sigma$ T

帛

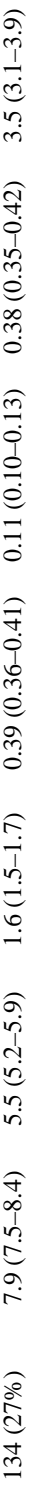

ํㅠㄹ

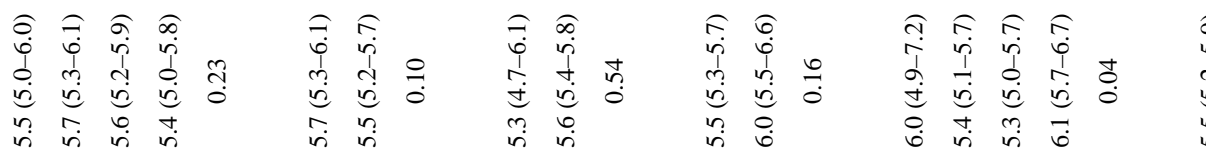

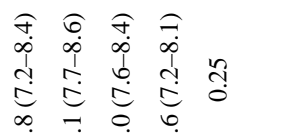

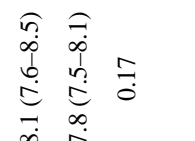

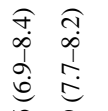

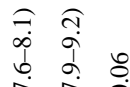

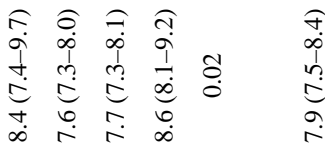

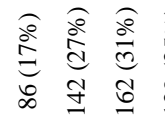

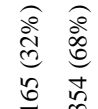

$\begin{array}{cc}c & 0 \\ 0 & 0 \\ 0 & 0 \\ 0 & 0 \\ \text { nn } & 0 \\ 0 & 0\end{array}$

$\begin{array}{cc}0 & 0 \\ \infty & 0 \\ \infty & d \\ n & 0 \\ y & 0\end{array}$

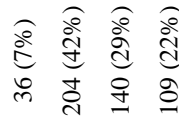

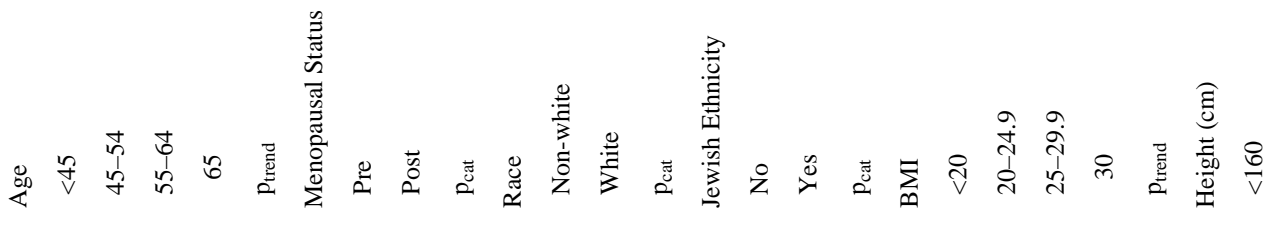




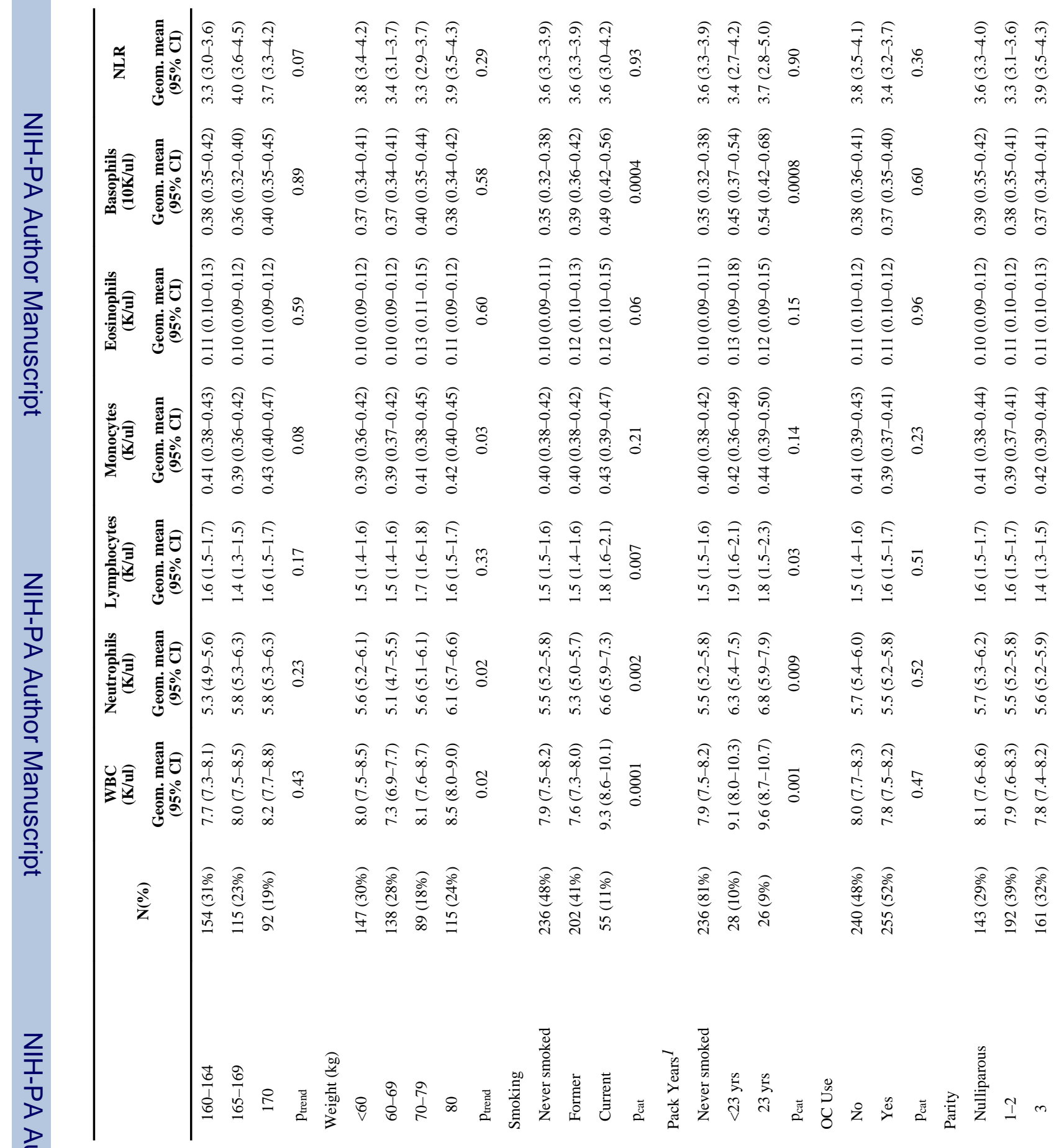




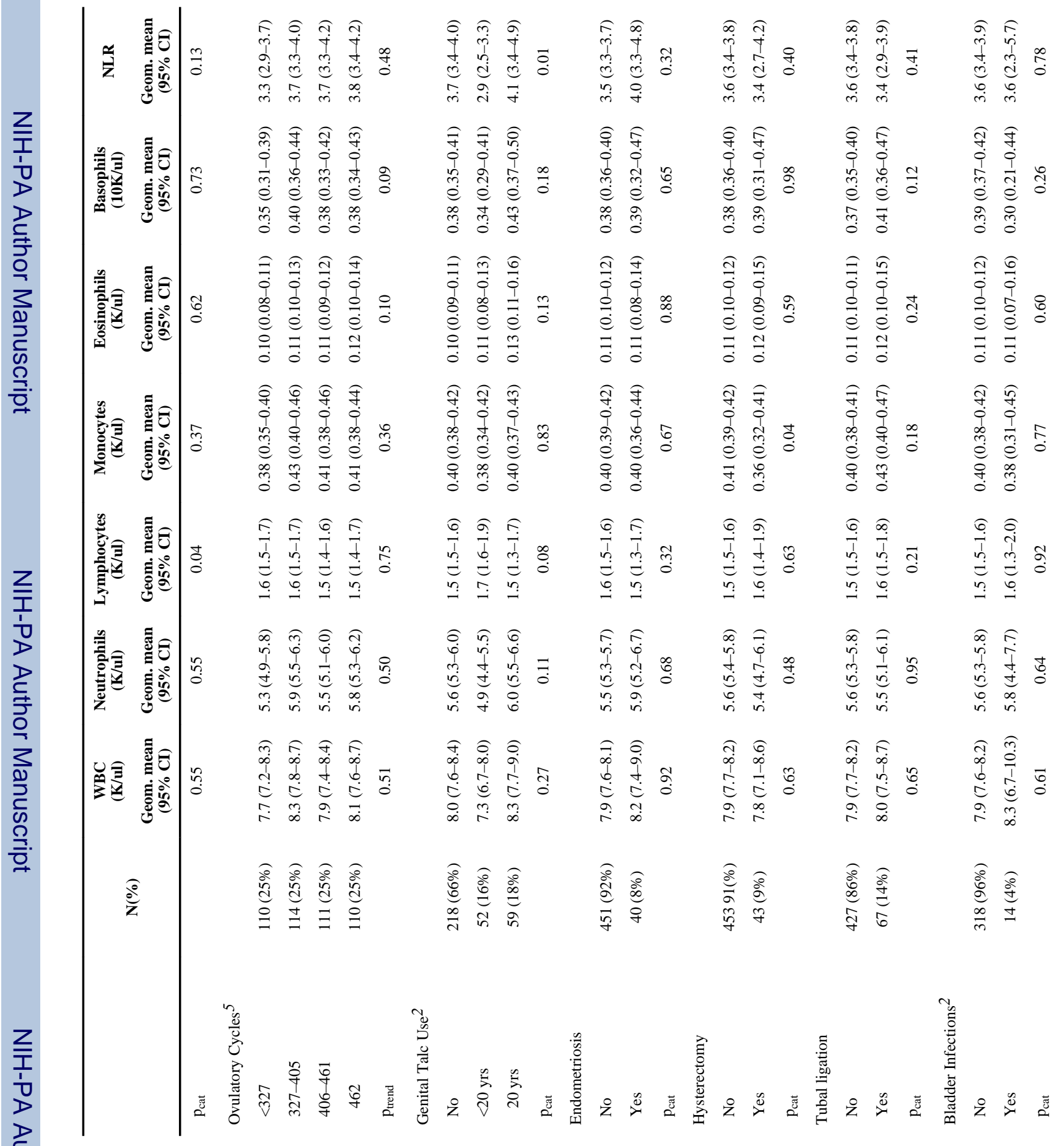




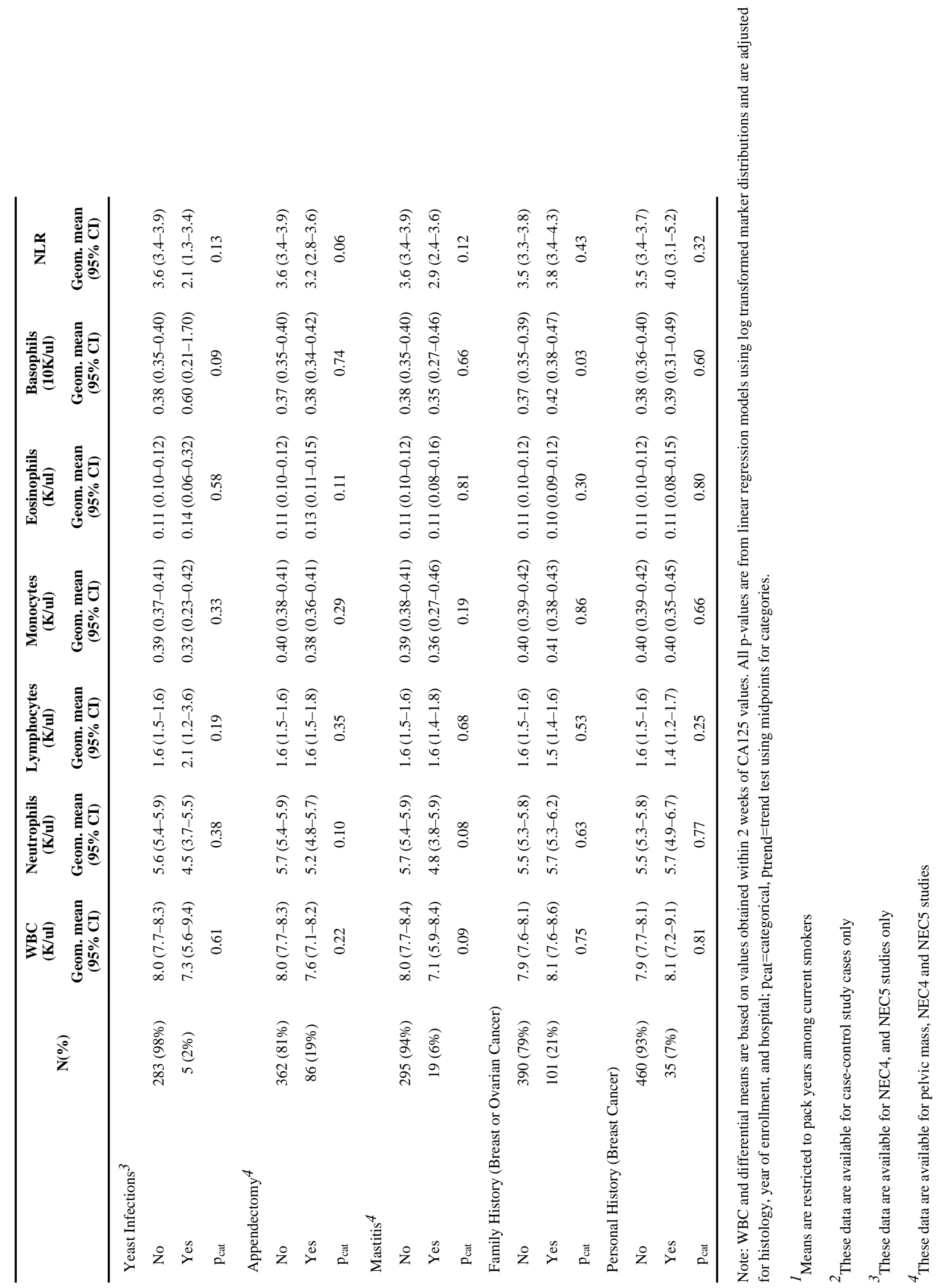




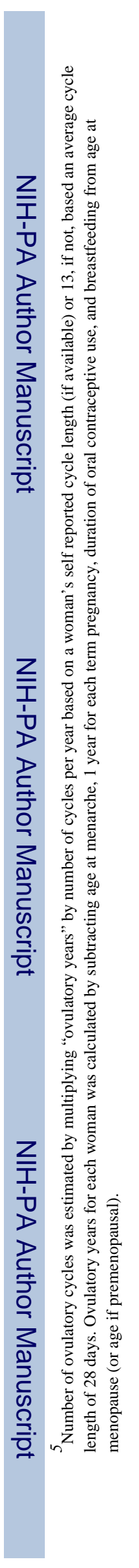

Gynecol Oncol. Author manuscript; available in PMC 2015 March 01. 


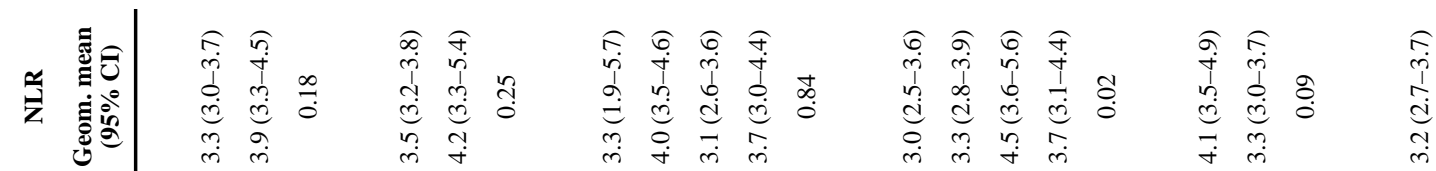

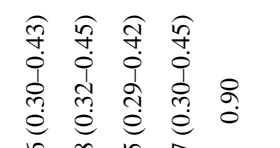

$\begin{array}{cc}\widehat{a} & \widehat{?} \\ 0 & 0 \\ 0 & 1 \\ 0 & 0\end{array}$

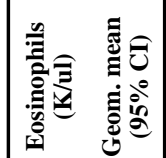

$\begin{array}{ll}1 & 1 \\ 0 & 0 \\ 0 & 0 \\ 0 & 0\end{array}$

궁

\begin{tabular}{cc} 
Iิ & \multicolumn{2}{c}{$c$} \\
0 & 0 \\
0 & 1 \\
0 & 0
\end{tabular}

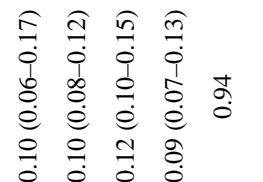

㐘

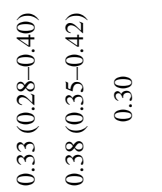

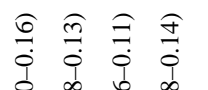

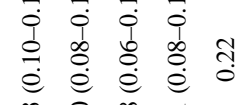

$\rightarrow \circ \infty$

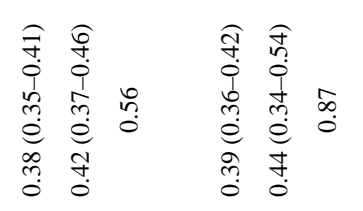

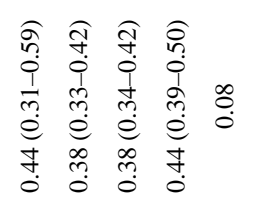

$\widehat{\exists} \widehat{\exists} \widehat{\sigma} \widehat{\sigma}$

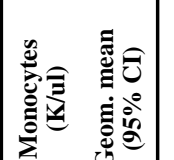

高

ะิ

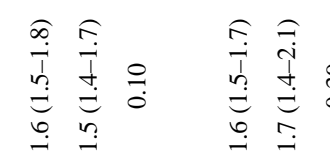

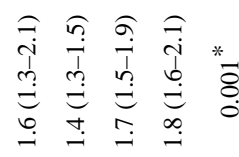

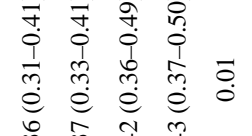

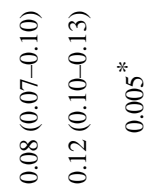

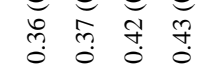

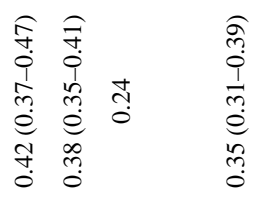

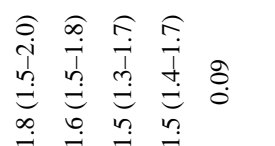

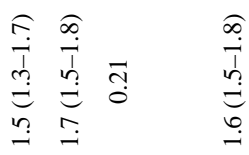

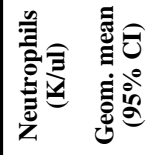

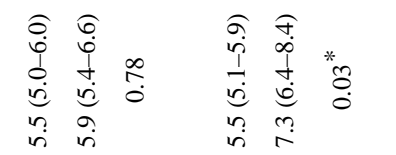

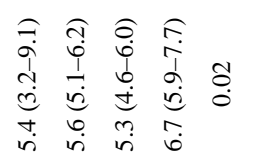

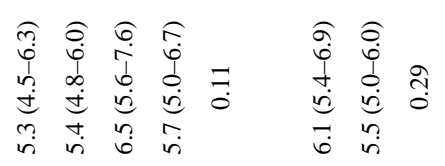

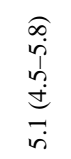

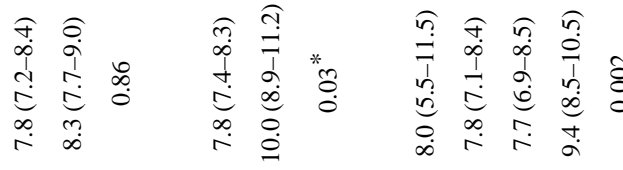

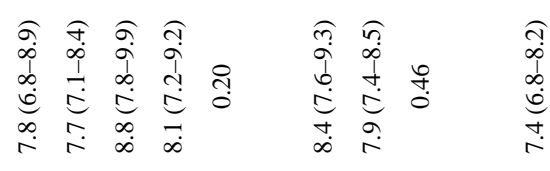

$\frac{2}{2} \mathrm{z}$

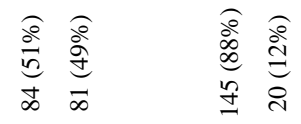

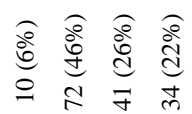

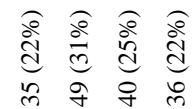

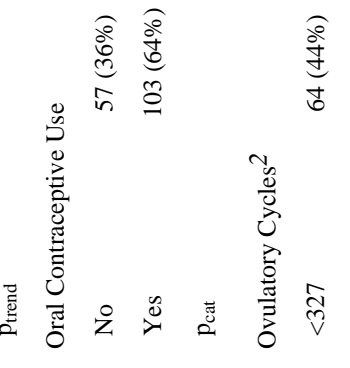

Gynecol Oncol. Author manuscript; available in PMC 2015 March 01. 


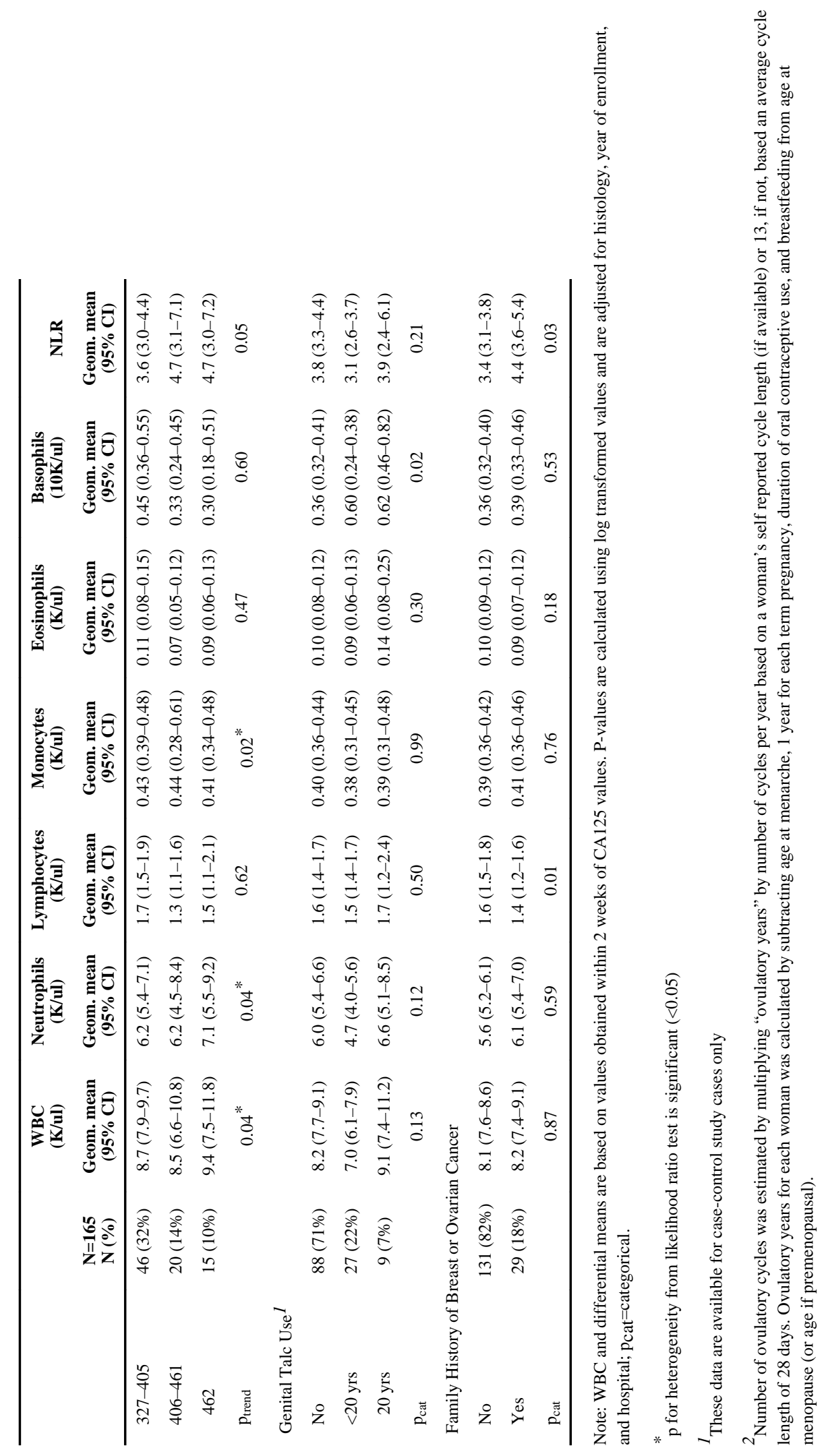




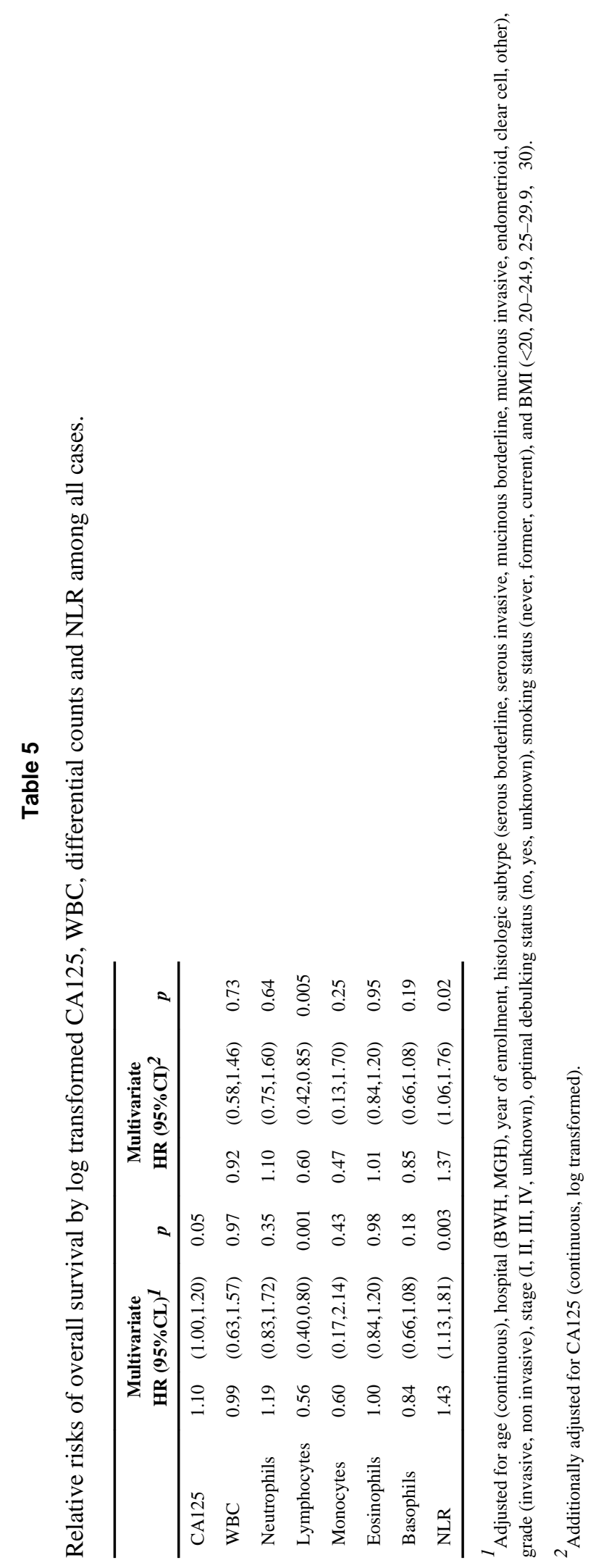

Gynecol Oncol. Author manuscript; available in PMC 2015 March 01. 